\title{
ELECTRON CLOUD IN THE FERMILAB BOOSTER
}

\author{
K.Y. Ng \\ Fermilab, P.O. Box 500, Batavia, IL 60510
}

\begin{abstract}
submitted to
22nd Particle Accelerator Conference 07 (PAC'07)

Albuquerque, New Mexico
\end{abstract}

June 25-29, 2007 


\title{
ELECTRON CLOUD IN THE FERMILAB BOOSTER
}

\author{
K.Y. Ng, ${ }^{*}$ Fermilab,${ }^{\dagger}$ Batavia, IL 60510, USA
}

\section{Abstract}

Simulations of the Fermilab Booster reveal a substantial electron-cloud buildup both inside the unshielded combined-function magnets and the beam pipes joining the magnets, when the second-emission yield (SEY) is larger than $\sim 1.6$. The implication of the electron-cloud effects on space charge and collective instabilities of the beam is discussed.

\section{STABILITY CONTOURS}

Following the analytic solution of Métral and Ruggiero, [1] we computed the stability contour of the Fermilab Booster beam near injection including space charge and octupole tune spread. The dashed curve in Fig. 1 is the stability contour in the complex coherent-tune-shift plane having an octupole tune spread \pm 0.05 with space charge turned off. The region under/above the contour implies stability/instability. As space charge is turned on, the stability contour becomes the solid curve. The Booster has a circumference of $2 \pi R=474.2 \mathrm{~m}$, composing of $84 \mathrm{rf}$ buckets. The Booster bunch is of intensity $N_{b}=6 \times 10^{10}$ at $1.40 \mathrm{GeV}$ (near injection), betatron tunes $\nu_{x, y}=6.7 / 6.8$, normalized rms emittance $2.0 \pi \mathrm{mm}-\mathrm{mr}$, and rms length $\sigma_{z}=0.70 \mathrm{~m}$, with maximum space charge tune shift $\Delta \nu_{\max }^{\mathrm{spch}} \sim 0.60$. In the derivation, coasting beam is assumed, but the peak current has been used. Now the stability region becomes much wider as a result of the large space-charge tune spread. Unfortunately, this wide stable area has been shifted far far away from center of the plot as a result of the large incoherent tune shift. The inductive part of the vacuum chamber impedance, which is usually small, must be extraordinary large to be under the contour in order to stabilize the beam.

\section{SPACE-CHARGE TUNE SHIFT}

The code POSINST [2] is employed to study electron cloud buildup near injection. The Booster is made up of 24 combined function F-magnets and 24 combined function D-magnets. In the simulations, the inside volume of the F-magnet where the beam resides is represented by a $13.0^{\prime \prime} \times 1.64^{\prime \prime}$ rectangular pipe with uniform magnetic field 0.084102 Tesla, while that of the D-magnet is represented

\footnotetext{
*ng@fnal.gov

$\dagger$ Operated by the U.S. Department of Energy, under contract with the Fermi Research Alliance, LLC.
}

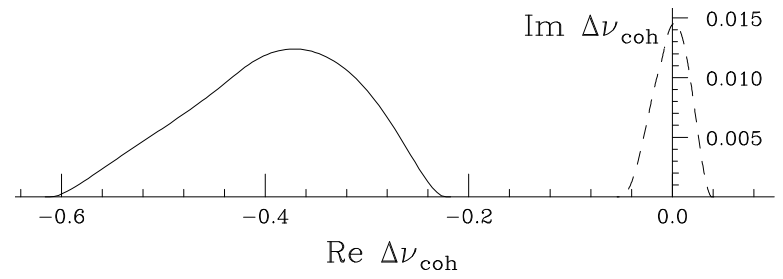

Figure 1: Stability contour from octupole with (solid) and without (dashes) space charge.

by a $12.0^{\prime \prime} \times 2.25^{\prime \prime}$ rectangular pipe with 0.071480 Tesla. According to the observed initial loss rate of $\sim 1.5 \%$ for the first 500 turns, beam loss to the surrounding per beam particle per meter is $6.49 \times 10^{-8}$, and each of these strayed particles is assumed to generate 100 electrons. They dominate over the electrons generated by collision with ions at the vacuum pressure of $1 \times 10^{-7}$ Torr. Figure 2 shows the electron density around one transverse $\sigma_{x, y}$ of the beam inside the F- and D-magnets for various SEY's. The bunch pattern has been taken to be 81 bunches plus 3 empty buckets. Thus the density dips in the plots correspond to the ends of revolution turns. We see that saturation is reached in the $D$-magnet when $S E Y \geq 1.5$, while it requires a $S E Y \geq 1.9$ to have saturation in the F-magnet. This may be due to the fact that the vertical gap of the D-magnet is much bigger and can therefore trap more electrons. The same simulations were performed for the $168 \mathrm{~m}$ of $2.25^{\prime \prime}$ and $28.8 \mathrm{~m}$ of $4.25^{\prime \prime}$ circular stainless steel pipes joining the magnets. The re-
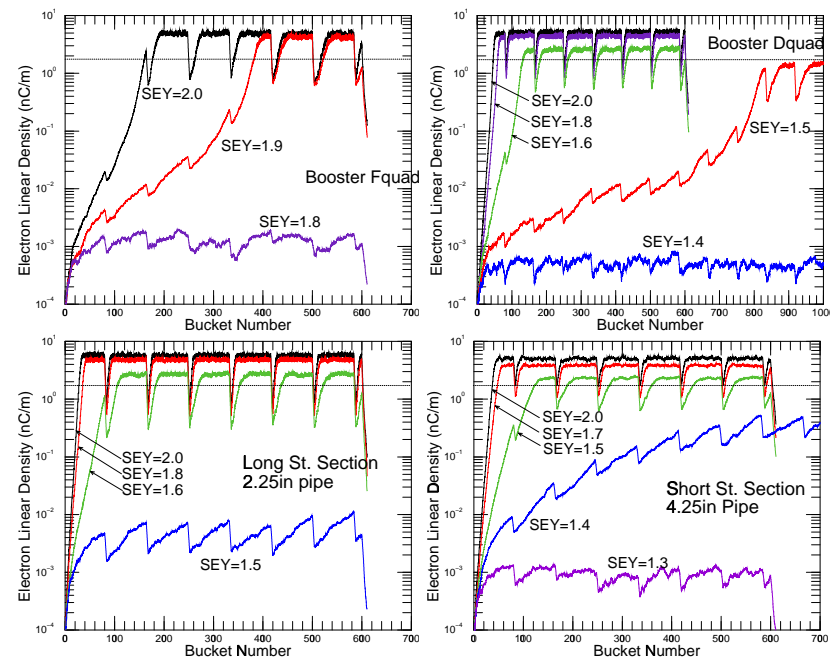

Figure 2: (Color) Electron cloud linear density inside an Fmagnet (top left), a D-magnet (top right), the $2.25^{\prime \prime}$ pipe in the long straight sections (bottom left), and the $4.25^{\prime \prime}$ pipe in the short straight sections (bottom right) for various values of SEY. The beam's average linear density is shown in dashes as a reference. 

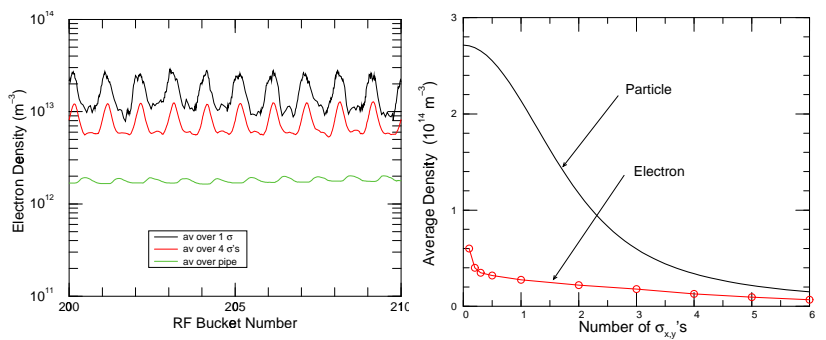

Figure 3: (Color) Left: Electron density inside a D-magnet with SEY $=1.6$ between rf buckets 200 and 210. Black, red, and green curves show electron density averaged over $1 \sigma_{x, y}$ of the beam, $4 \sigma_{x, y}$ 's, and the whole cross section of the magnet. Right: Particle density and electron density averaged over $n \sigma_{x, y}$ 's.

sults are shown in Fig. 2. Again the larger pipe appears to have the ability to trap more electrons. In any case, however, electron cloud reaches saturation when $\mathrm{SEY} \gtrsim 1.6$.

It is unfortunate that we have no knowledge of the SEY for magnet laminations. In below, we try to do the investigation using $\mathrm{SEY}=1.6$, implying that electron cloud buildup will saturate in the round pipes and inside the Dmagnets, but not necessary in the F-magnets. We next look into the electron density near the beam in Fig. 3. Since the peak beam particle density is $\rho_{b}^{\mathrm{pk}}=2.72 \times 10^{14} \mathrm{~m}^{-3}$, the electron density appears to be very much smaller. However, the particle density decreases very rapidly away from the beam axis, but the electron density does not. For example, the ratio of electron density to particle density averaged over two $\sigma_{x, y}$ 's is 0.187 . The implication is that the cancellation of space charge of the beam may be $\sim 18.7 \%$, which is rather appreciable. The inductive tune shift of the beam particle in the electron cloud can also be estimated by assuming a uniform electron density, giving

$$
\Delta \nu_{\mathrm{cl}}=\frac{\pi \rho_{e} r_{p} R^{2}}{\gamma \beta^{2} \nu_{y}},
$$

where $r_{p}=1.535 \times 10^{-18} \mathrm{~m}$ is the classical proton radius, and $\gamma$ and $\beta$ are relativistic factors. The beam particles reside mostly within two $\sigma_{x, y}$ 's of the bi-Gaussian distribution. We therefore read off $\rho_{e}=2.2 \times 10^{13} \mathrm{~m}^{-3}$ from Fig. 3 as the electron density averaged over two $\sigma_{x, y}$ 's. This gives $\Delta \nu_{\mathrm{cl}}=0.11$, which is $18.0 \%$ of the maximum space-charge tune shift $\Delta \nu_{\max }^{\mathrm{spch}}=0.60$. The tune depression of an intense Booster beam as well as the inductive part of the magnet laminations and connecting beam pipe has been measured and computed [4] and is found to be $\sim 0.04$. Thus, in total, at most $\sim 25 \%$ of the space charge will be canceled by electron cloud and inductive walls. As is shown in the stability contours of Fig. 1, there is still no possibility for the beam's impedance to be inside the stable region.

\section{COLLECTIVE INSTABILITIES}

The effects of the electron cloud can be modeled by a short range wake. In Heifets derivation, this wake is [3]

$$
W_{1}(z)=\frac{8 Z_{0} \rho_{e} \omega_{e} R}{(1+p) \lambda_{b}^{\mathrm{pk}}} W_{\mathrm{eff}}(z),
$$

where $p=\sigma_{y} / \sigma_{x}$ is the aspect ratio of the particle beam with peak linear density $\lambda_{b}^{\mathrm{pk}}=N_{b} / \sqrt{2 \pi} \sigma_{z}$, where $\sigma_{z}$ is the rms bunch length. The effective wake $W_{\text {eff }}(z)$ is depicted in Fig. 4 for various ratios of the rms spread of the cloud $\Sigma_{x, y}$ to that of the beam $\sigma_{x, y}$. The transverse impedance, computed by performing a Fourier transform, is depicted in Fig. 4, where an average electron density of $\rho_{e}=1 \times 10^{13} \mathrm{~m}^{-3}$ in the vicinity of the beam has been assumed. Alongside, we have also plotted the transverse impedance of the 48 laminated magnets. We see that the impedance arising from the electron cloud is mostly dominated by a resonance near the electron bounce frequency $\omega_{e} / 2 \pi$ and is much larger than that from the magnets below $\sim 140 \mathrm{MHz}$. (The electron bounce frequency increases during ramping as a result of bunch-length and beam-size shrinkings, and so does the position of the resonance frequency in the cloud impedance.) This is to be expected, because a larger inductive impedance at low frequencies needed to partially cancel more space charge of the beam will unavoidably bring about large $\operatorname{Re} Z_{1}^{\perp}$ and thus severe transverse head-tail instabilities and transverse microwave instabilities to the Booster beam. Since these rather large instabilities have not been observed, it is possible that the SEY's of the magnet laminations and the adjoining beam pipes are much smaller, for example, $\lesssim 1.3$, so that electron cloud does not accumulate around the beam. For example, the electron cloud effects will become minimal when the cloud density is reduced to below $1 \times 10^{12} \mathrm{~m}^{-3}$.
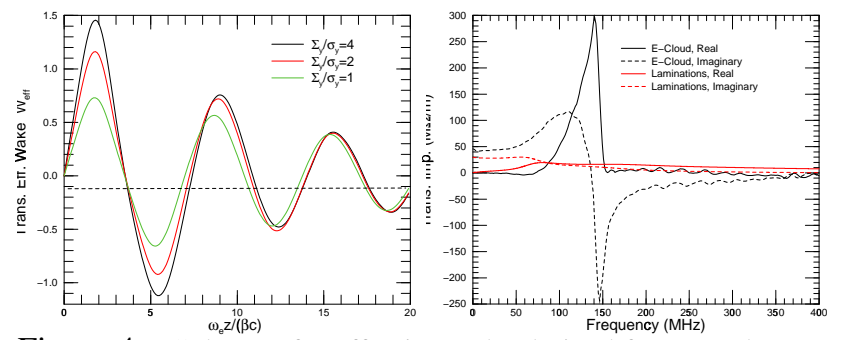

Figure 4: (Color) Left: Effective wake derived from an electron cloud around a round beam, where $\Sigma_{y}$ and $\sigma_{y}$ are the vertical rms radii of the cloud and beam, respectively. Right: Real and imaginary parts of the transverse impedances arising from electron cloud in the Booster near injection, resonating strongly near the electron bounce angular frequency $\omega_{e}$ with $\Sigma_{y} / \sigma_{y}=2$. The much smaller transverse impedance coming from the magnet laminations is also shown for comparison. 


\section{EFFECT OF BUNCHING}

In the discussion of stability contour earlier a coasting Booster beam has been assumed. The situation of a bunched beam can be very different. This is because there will be many more particles having smaller space-charge tune shifts, for example those away from the longitudinal center. Here we will study the simpler problem concerning the distribution of space-charge tune shifts of the particles inside a bunch, which can also shed some light on the shape of the corresponding stability contour.

The distribution of space-charge tune shift in a coasting beam with circular cross section and bi-Gaussian distributed, $f_{2 D}\left(\Delta \nu^{\mathrm{spch}} / \Delta \nu_{\max }^{\mathrm{spch}}\right)$, is depicted in dashes in Fig. 5. It is skewed towards higher values, with $\left\langle\Delta \nu^{\mathrm{spch}}\right\rangle / \Delta \nu_{\max }^{\mathrm{spch}}=0.6334$. The distribution is essentially zero when $\Delta \nu^{\text {spch }} / \Delta \nu_{\max }^{\text {spch }}<0.15$. This curve closely resembles the stability contour in Fig. 1(a). In fact, they should be closely related. For a bunch, however, the space-charge tune shift distribution can be very different because the particles near the two ends have rather small space-charge tune shifts. The tune shift distribution for the whole bunch can be readily derived to be [5]

$$
F_{3 D}\left(\frac{\Delta \nu^{\mathrm{spch}}}{\Delta \nu_{\max }^{\mathrm{spch}}}\right)=\int_{-z}^{z} f_{2 D}\left(\frac{\Delta \nu^{\mathrm{spch}}}{\Delta \nu_{\max }^{\mathrm{spch}}} \frac{\lambda_{b}(0)}{\lambda_{b}\left(z^{\prime}\right)}\right) \lambda_{b}(0) d z^{\prime},
$$

where $\lambda_{b}(z)$ is the linear density and the limits of integration $\pm z$ are given by $\Delta \nu^{\text {spch }} \frac{\lambda_{b}(0)}{\lambda_{b}(z)}=1$. These 3D distributions for some common linear distributions are depicted in Fig. 5. They show that there are plenty of particles with space-charge tune shift close to zero, especially when the longitudinal linear density has longer tails. A longitudinal Gaussian distribution may have been too ideal, but the cosine-square distribution is rather realistic. We expect the stability contour for a bunch behaves similarly. As a result, beam stability can be attained provided that there is some

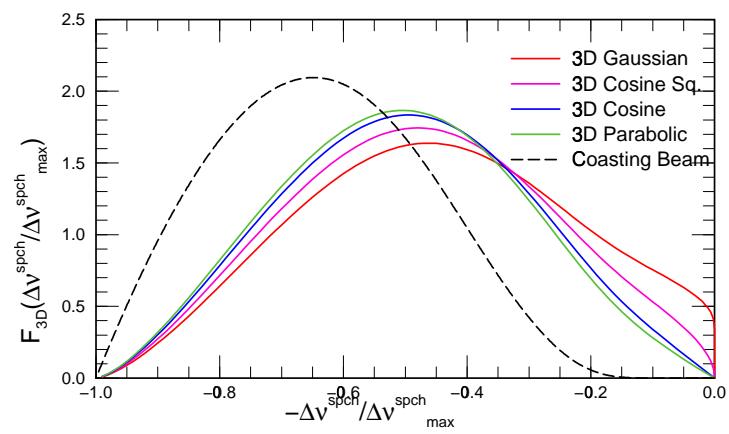

Figure 5: (Color) Plots of distribution in space-charge tune shift for a bi-Gaussian round bunch with longitudinal Gaussian, cosine square, cosine, or parabolic distribution. The distribution of the unbunched beam is also shown for comparison. reasonable inductive impedance, some extra tune spread from octupoles, and the $\left|\operatorname{Re} Z_{1}^{\perp}\right|$ is not too big, while electron cloud need not play an important role.

\section{CONCLUSION}

We studied the stability contour of the Booster beam in the presence of space charge and octupoles, and found that the electron cloud buildup with $\mathrm{SEY}=1.6$ is hardly enough to neutralize the space charge and stabilize the beam. The electron cloud, on the other hand, will bring about strong $\operatorname{Re} Z_{1}^{\perp}$ near the electron bounce frequency of $\sim 140 \mathrm{MHz}$ at injection, leading to undesirable transverse collective instabilities. Since these instabilities have not been observed, either the electron cloud buildup is much smaller due to a smaller SEY of the laminated magnets, or the derived wake is incorrect or it behaves differently from the usual wake of the vacuum chamber discontinuities.

We have also studied the space-charge tune shift distribution when the beam is bunched. Since there are many more low space-charge tune shifted particles, the tune shift distribution is now skewed back towards the zero tune shift side. We believe the stability contour for a bunched beam will behave in the same way; i.e., there will be ample stable region under the stability contour close to the origin of the complex coherent-tune-shift space. As a result, a small amount of inductive impedance together with some octupole tune spread will be able to stabilize the Booster beam, provided that $\left|\mathcal{R e} Z_{1}^{\perp}\right|$ is not too large. This paper serves as an extract of the more detailed version of Ref. [5].

\section{REFERENCES}

[1] E. Métral and F. Ruggiero, Stability diagrams for Landau damping with two-dimensional betatron tune spread from both octupoles and non-linear space charge, CERN-AB2004-025 (ABP), 2004.

[2] M.A. Furman and G.R. Lambertson, The Electron-Cloud Instability in the Arcs of the PEP-II Positron Ring, Proc. Int. Workshop on Multibunch Instabilities in Future Electron and Positron Accelerators (MBI-97), ed. Y.H. Chin, KEK, Tsukuba, Japan, July 15-18, 1997.

[3] S. Heifets, Wake field of the e-cloud, Proc. 23rd Advanced ICFA Beam Dynamics Workshop on High Luminosity $e^{+} e^{-}$ Collider, Ithaca, NY, October 15-19, 2001; SLAC-PUB9025, 2001.

[4] Xiaobiao Huang, Beam Diagnosis and Lattice Modeling of the Fermilab Booster, $\mathrm{PhD}$ thesis, Indiana University, 2005;

[5] K.Y. Ng, Electron-Cloud and Space-Charge Effects in the Fermilab Booster, Fermilab Report FN-0803-AD, 2007. 